\title{
OPEN Marangoni effect inspired robotic self-propulsion over a water surface using a flow-imbibition-powered microfluidic pump
}

\begin{abstract}
Bokeon Kwak, Soyoung Choi, Jiyeon Maeng \& Joonbum Bae ${ }^{\bowtie}$
Certain aquatic insects rapidly traverse water by secreting surfactants that exploit the Marangoni effect, inspiring the development of many self-propulsion systems. In this research, to demonstrate a new way of delivering liquid fuel to a water surface for Marangoni propulsion, a microfluidic pump driven by the flow-imbibition by a porous medium was integrated to create a novel self-propelling robot. After triggered by a small magnet, the liquid fuel stored in a microchannel is autonomously transported to an outlet in a mechanically tunable manner. We also comprehensively analyzed the effects of various design parameters on the robot's locomotory behavior. It was shown that the traveled distance, energy density of fuel, operation time, and motion directionality were tunable by adjusting porous media, nozzle diameter, keel-extrusion, and the distance between the nozzle and water surface. The utilization of a microfluidic device in bioinspired robot is expected to bring out new possibilities in future development of self-propulsion system.
\end{abstract}

The characteristics of bio-locomotion on the air/water interface is different from those of other environments; surface tension plays an important role. Arthropods such as the rove beetles Microvelia and Stenus are both supported by water surface tension, and rapidly (up to $70 \mathrm{~cm} / \mathrm{s}$ ) skim over the water surface as shown in Fig. 1A by secreting chemicals that momentarily reduce the surface tension to the rear ${ }^{1-3}$. Such locomotion is commonly known as Marangoni propulsion, caused by interfacial flow toward increasing surface tension, and has been widely used in the development of self-propelling systems (Table 1$)^{4-6}$. Alcohol commonly serves as the propellant; however, other organic compounds are sometimes used. Various methods of propellant delivery to the water surface have been described, of which spontaneous transfer via a propellant-infused matrix is the most common $^{7-14}$, although thrust is sometimes generated by dispensing alcohol droplets ${ }^{15-18}$. Spontaneous release of alcohol from a reservoir is also employed ${ }^{19-23}$. Other interesting mechanisms include vapor diffusion ${ }^{24}$, alcohol condensation $^{25}$, depolymerization reactions ${ }^{26}$, and photothermally induced fuel release ${ }^{27}$.

Many such systems trigger immediate propulsion, and some studies have sought to control the timing of thrust generation (Table 1$)^{16-18,23,27}$. However, the actuators increase weight ${ }^{16,17}$, and speed is slowed at certain $\mathrm{pH}$ values ${ }^{23}$. Also, some triggers did not act instantly ${ }^{18,27}$. An additional problem associated with droplet-based dispensing is the need for a vertical vessel filled with propellant that flows out under its own weight ${ }^{15-18}$. A fully loaded vessel concentrates force on the rear of the robot and increases the risk of submergence during propulsion.

Here, we develop a new Marangoni propulsion-based system: a robot that travels on water featuring a capillary pressure-driven microfluidic pump as shown in Fig. 1B. Capillary pressure induced by liquid imbibition by a porous medium has previously been exploited to drive paper-based microfluidic devices ${ }^{28-30}$. However, such pump was redesigned to develop our new self-propulsion system. We use magnetic clamping to trigger the formation of alcohol droplets at required times. Passive actuation is required, and motion is instantaneous, $\mathrm{pH}$ independent, and repeatable. The proposed pump is comprehensively analyzed by adjusting its outlet diameter and embedded porous medium. We also performed a full locomotion analysis and compared the robot to previous systems. Given the recent advances in soft machines featuring autonomous flow regulation ${ }^{31,32}$, microfluidic self-propulsion systems will find many applications. 


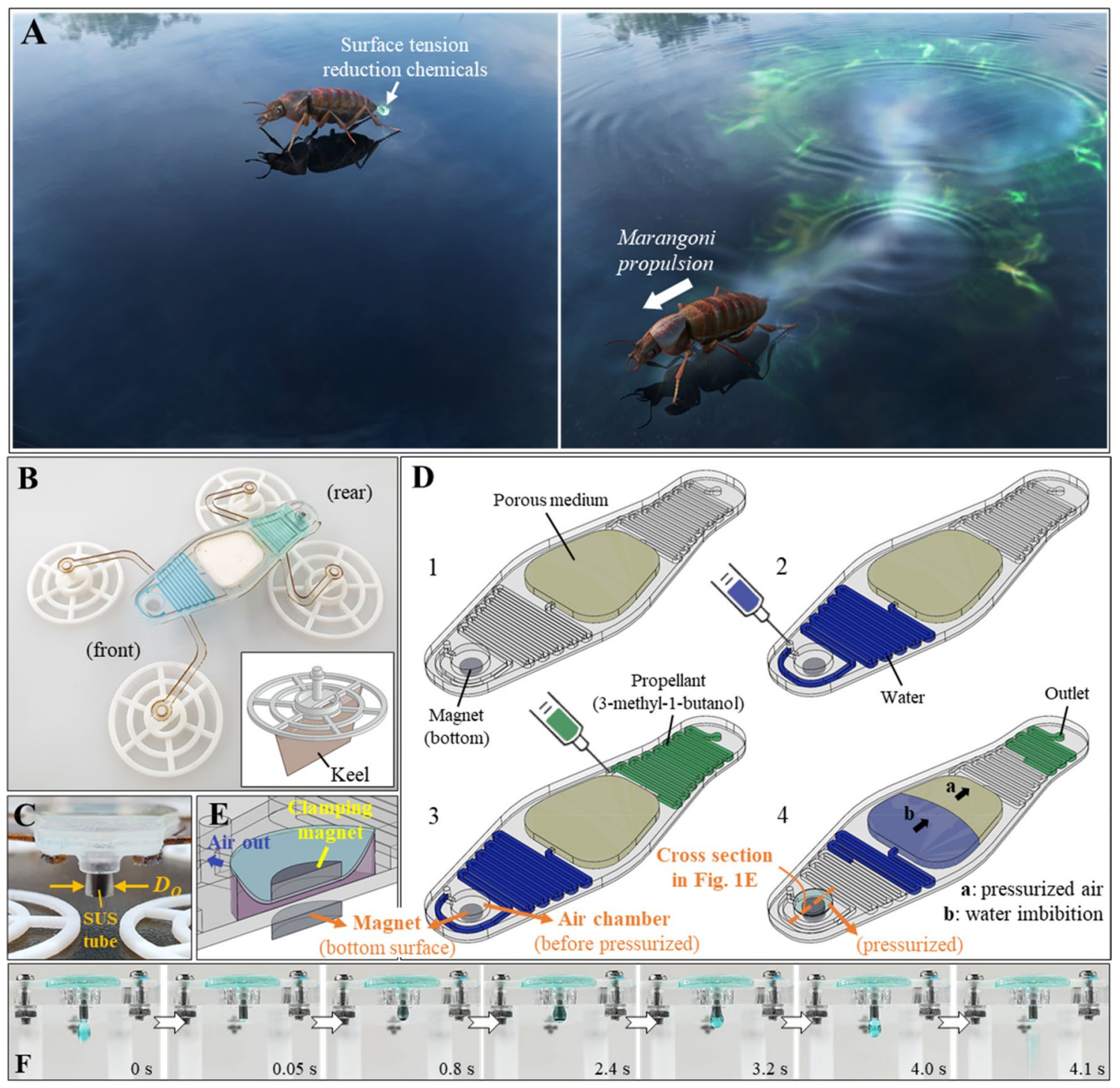

Figure 1. Structure and working principle of the robot exploiting Marangoni propulsion. (A) Secretion of chemicals that reduce surface tension. (B) The proposed self-propelling robot (bottom-right corner: a keelextruded footpad). (C) The rear side of the robot. (D) The preparative steps prior to pump activation. (E) A cross-section of the air-filled chamber of D-(step 4). (F) Alcohol droplet formation during pump operation $\left(D_{O}=1.5 \mathrm{~mm}\right)$.

\begin{tabular}{|l|l|l|}
\hline Mechanism & Trigger & Refs. \\
\hline \multirow{2}{*}{ Propellant transfer from soaked gel, droplet, pellet, or other forms of matrix } & No & $7-14$ \\
\hline \multirow{2}{*}{ Spontaneous release from a reservoir } & No & 15 \\
\cline { 2 - 3 } & Servo motor & 16,17 \\
\cline { 2 - 3 } & Water droplet & 18 \\
\hline Vapor diffusion & No & $19-22$ \\
\hline Condensation & Alkaline solution & 23 \\
\hline Depolymerization reaction & No & 24 \\
\hline Photo-thermally induced alcohol release & No & 25 \\
\hline Self-powered microfluidic pump & No & 26 \\
\hline
\end{tabular}

Table 1. State-of-the-art in self-propulsion systems driven by Marangoni effect. Here, no trigger means the self-propulsion started immediately when it was placed on liquid/air interface, or right after filling with propellant. 


\begin{tabular}{|c|c|c|c|}
\hline Symbols & Definitions & Symbols & Definitions \\
\hline$D_{O}$ & Nozzle diameter & $\sigma_{a}$ & Surface tension of alcohol \\
\hline FP & Filter paper & $\sigma_{w}$ & Surface tension of water \\
\hline $\mathrm{CP}$ & Cellulose powder & BTB & Bromothymol blue \\
\hline$h_{f}$ & Footpad height & $R_{c}$ & Ring trace radius \\
\hline$Q_{w}$ & Average flow rate of water & $S_{d}$ & Traveled distance \\
\hline$Q_{a}$ & Average flow rate of alcohol & $\varepsilon_{K, \text { max }}$ & Energy density of fuel \\
\hline$P_{c}$ & Capillary pressure & $T_{m}$ & Duration of motion \\
\hline$\phi$ & Porosity & $C_{\theta}$ & Trajectory characteristic \\
\hline$r_{m}$ & Mean pore radius & $m_{\text {robot }}$ & Mass of the robot \\
\hline$\theta$ & Contact angle & $m_{\text {fuel }}$ & Mass of the fuel \\
\hline$k$ & Permeability & $V_{\max }$ & Maximum propulsion speed \\
\hline$V_{a}$ & Droplet volume of alcohol & $\Delta T$ & Sampling time in $C_{\theta}$ \\
\hline$M_{a}$ & Total volume of injected alcohol & $\theta_{i}$ & Heading angle change $(i=1,2, \ldots)$ \\
\hline$T_{\text {drop }}$ & Time between breakup of two consecutive droplets & $T_{a w}$ & Time between two consecutive alcohol-water surface contact \\
\hline$N_{\text {drop }}$ & Total number of droplets generated & $h_{a}$ & Oscillation of alcohol droplet height \\
\hline$g$ & Gravitational acceleration constant & $W e_{\max }$ & Maximum Weber number \\
\hline$\psi$ & Harkins-Brown (H-B) correction factor & $R e_{\max }$ & Maximum Reynolds number \\
\hline$\rho_{a}$ & Density of alcohol & $w_{f}$ & Foot width (or, characteristic length) \\
\hline$\rho_{w}$ & Density of water & fps & Frames per second \\
\hline$\mu_{w}$ & Kinematic viscosity of water & $n$ & The number of trials \\
\hline
\end{tabular}

Table 2. The definitions of all the parameters and acronyms used on the main text.

\section{Results}

The working principle of the robot. The robot features four footpads allowing it to float by exploiting surface tension (Fig. 1B) ${ }^{33}$. The rear footpads are larger than the front footpads because the alcohol droplets reduce the surface tension at the rear. The maximal supported force by all the four footpads is about threefold that of the robot including fuel mass (refer Supplementary Note: Maximal supporting force). A footpad can (optionally) feature a thin keel (Fig. 1B, bottom-right corner) for directional motion (refer Supplementary Note: Drag asymmetry). Microchannels filled with blue-dyed water and green-dyed alcohol are located at the front and rear of the pump, respectively. A stainless-steel (SUS) tube of outer diameter $D_{O}$ is located to the rear of the pump (Fig. 1C) and generates alcohol droplets when the pump is operating (Fig. 1D). A porous medium is placed in the center of the pump and the top sealed (step 1). Water is injected into the channel (step 2), followed by alcohol (step 3). We used 3-methyl-1-butanol (3Me1Bu) because the Marangoni effect thereof is prolonged ${ }^{34}$. It is important to ensure that the apices of the injected liquid streams do not contact the porous medium. Note that a magnet is readily attached to the bottom surface of the air chamber before activating the pump. Finally, another magnet (i.e. clamping magnet) is manually placed on the air chamber to trigger water imbibition (step 4); the attractive force between the two magnets instantly pressurizes the air chamber and its cross section is given in Fig. 1E. During water imbibition, air within the porous medium becomes pressurized and pushes the alcohol out via the nozzle (Fig. 1F; Supplementary Videos S1-S4). The alcohol droplets fall near the rear footpads and the robot moves by Marangoni effect. The pump operates until all the water is fully imbibed by the porous medium. By comparing the pumping speed of propellant and the successful rate of magnetic clamping (refer Supplementary Note: Porous media selection and Table S1) $)^{35,36}$, two types of porous media (MN 617 filter paper [FP] and cellulose powder [CP]) were selected and used in the rest of the experiments. Please refer Table 2 for the definitions of parameters and acronyms used in this article.

Characteristics of the proposed pump. Dripping dynamics are affected by the liquid per se, the outlet nozzle diameter, and the flow rate. We tested nozzles of diameter $D_{O}=1.0,1.5,2.2$, and $3.2 \mathrm{~mm}$, and adjusted the flow rate by varying the porous medium. We measured droplet counts, dripping intervals, and periodicity. The volumes of injected water and alcohol were around $0.13 \mathrm{~mL}$ as summarized in Supplementary Table S2.

The key parameters were porous medium type and nozzle diameter. As it will be shown in locomotion analysis, two additional parameters (i.e. footpad height and keel-extrusion) will be considered. Here, Fig. 2A shows how the porous medium types affect droplet breakup with other properties such as nozzle diameter and footpad height. Each porous medium exhibit different properties as summarized in Table 3, and their identification methods are described in Supplementary Notes: Capillary pressure measurement ${ }^{37}$, Porosity measurement, and Contact angle and mean pore radius. Average flow rate of water $\left(Q_{w}\right)$ and the resultant alcohol flow rate $\left(Q_{a}\right)$ depend on porous medium as suggested in Fig. 2B; the flow rates were almost the same in different nozzle diameters (Supplementary Notes: Average flow rates) ${ }^{38}$. In this paper, the number of trials is denoted as $n$. Notably, $Q_{a}$ was about $80 \%$ of $Q_{w}$ for both FP and CP, likely attributable to (a very small extent of) air leakage during pumping, and pressure transmission loss from the frontal interface of the imbibed water to the alcohol. 

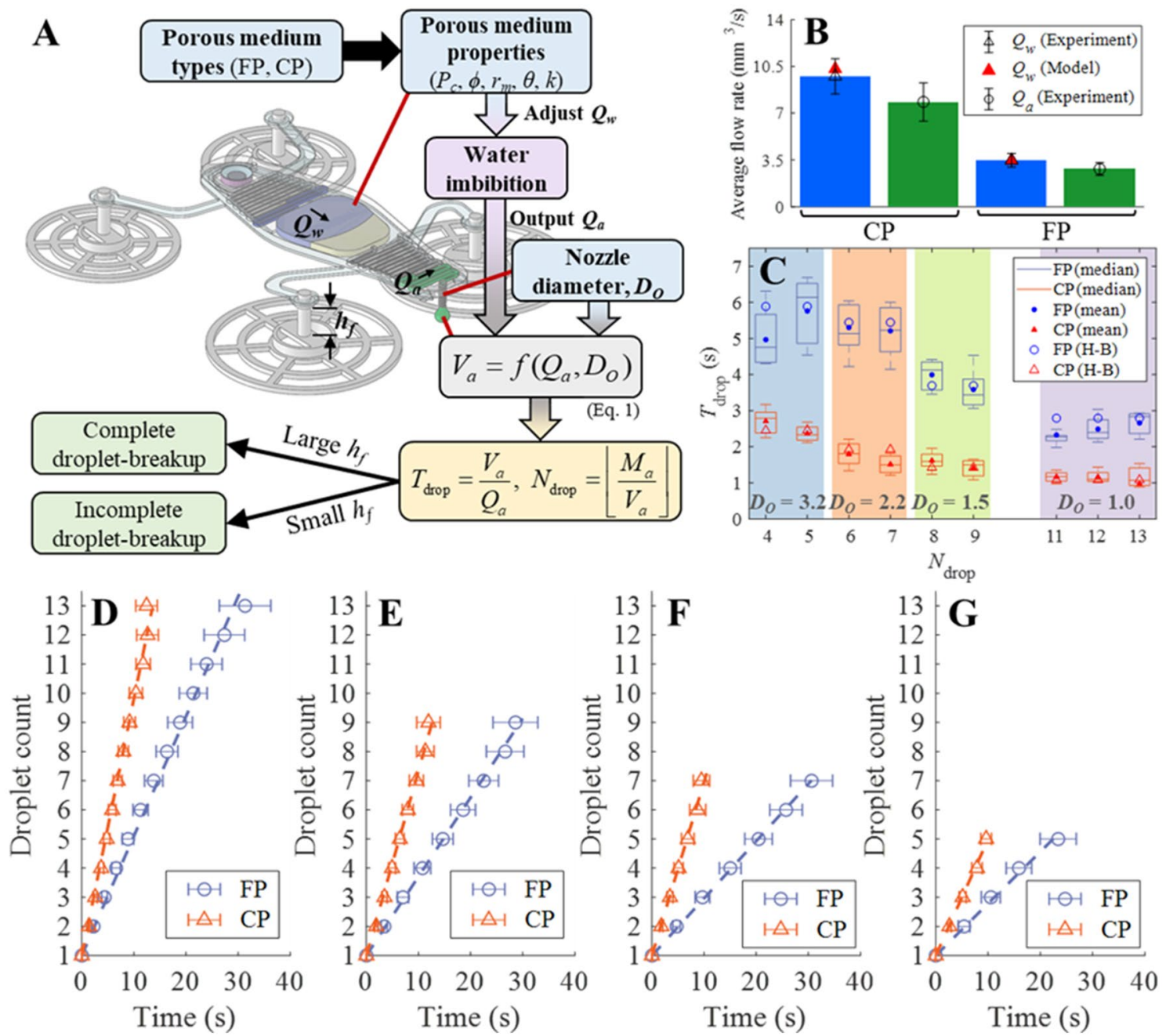

Figure 2. Analysis of alcohol dripping. (A) The relations from porous medium types to droplet breakup. Please refer Table 2 for the definitions of parameters and acronyms. (B) Average flow rates of water and alcohol $(n=5)$. (C) $T_{\text {drop }}$ vs. $N_{\text {drop }}$ during the pump operation where $D_{O}$ denotes the nozzle diameter in millimeter $(n=15)$. Theoretical $T_{\text {drop }}$ values incorporating the Harkins-Brown (H-B) correction factors are depicted by hollow circles. (D-G) The elapsed time of alcohol droplet breakup (D: $D_{O}=1.0$, E: $D_{O}=1.5, \mathbf{F}: D_{O}=2.2$, G: $\left.D_{O}=3.2 \mathrm{~mm}\right)$; dashed lines represent linear regression data $(n=15)$. Note that all the error bars denote standard deviations.

\begin{tabular}{|l|l|l|}
\hline Properties & Filter paper $(\mathbf{F P})$ & Cellulose powder $(\mathbf{C P})$ \\
\hline Capillary pressure $\left(P_{c}\right)$ & $0.93 \pm 0.14 \mathrm{kPa}$ & $4.90 \pm 0.58 \mathrm{kPa}$ \\
\hline Porosity $(\phi)$ & 0.6722 & 0.6529 \\
\hline Contact angle $(\theta)$ & $83.36 \pm 0.87 \mathrm{deg}$ & $66.77 \pm 2.53 \mathrm{deg}$ \\
\hline Mean pore radius $\left(r_{m}\right)$ & $14.14 \pm 2.67 \mu \mathrm{m}$ & $11.62 \pm 2.02 \mu \mathrm{m}$ \\
\hline Permeability $(k)$ & $8.9827 \times 10^{-12} \mathrm{~m}^{2}$ & $6.0008 \times 10^{-12} \mathrm{~m}^{2}$ \\
\hline
\end{tabular}

Table 3. Important properties of porous media in used.

Also, $Q_{w}$ is well explained by its model (see Supplementary Notes: Flow rate models for details) ${ }^{39-46}$. Both $Q_{a}$ and $D_{O}$ determine an alcohol droplet volume $\left(V_{a}\right)$ which found as:

$$
V_{a}=\psi\left[\frac{\pi D_{O} \sigma_{a}}{\rho_{a} g}-\frac{16 Q_{a}^{2}}{3 \pi D_{O}^{2} g}+11.334\left(\frac{Q_{a}^{2} D_{O}^{2} \sigma_{a}}{\rho_{a} g^{2}}\right)^{1 / 3}\right]
$$

where $g$ is gravitational acceleration constant, $\psi$ is Harkins-Brown (H-B) correction factor (see Supplementary Notes: Harkins-Brown correction factor $)^{47,48}$, and $\rho_{a}$ and $\sigma_{a}$ are density and surface tension of alcohol, 


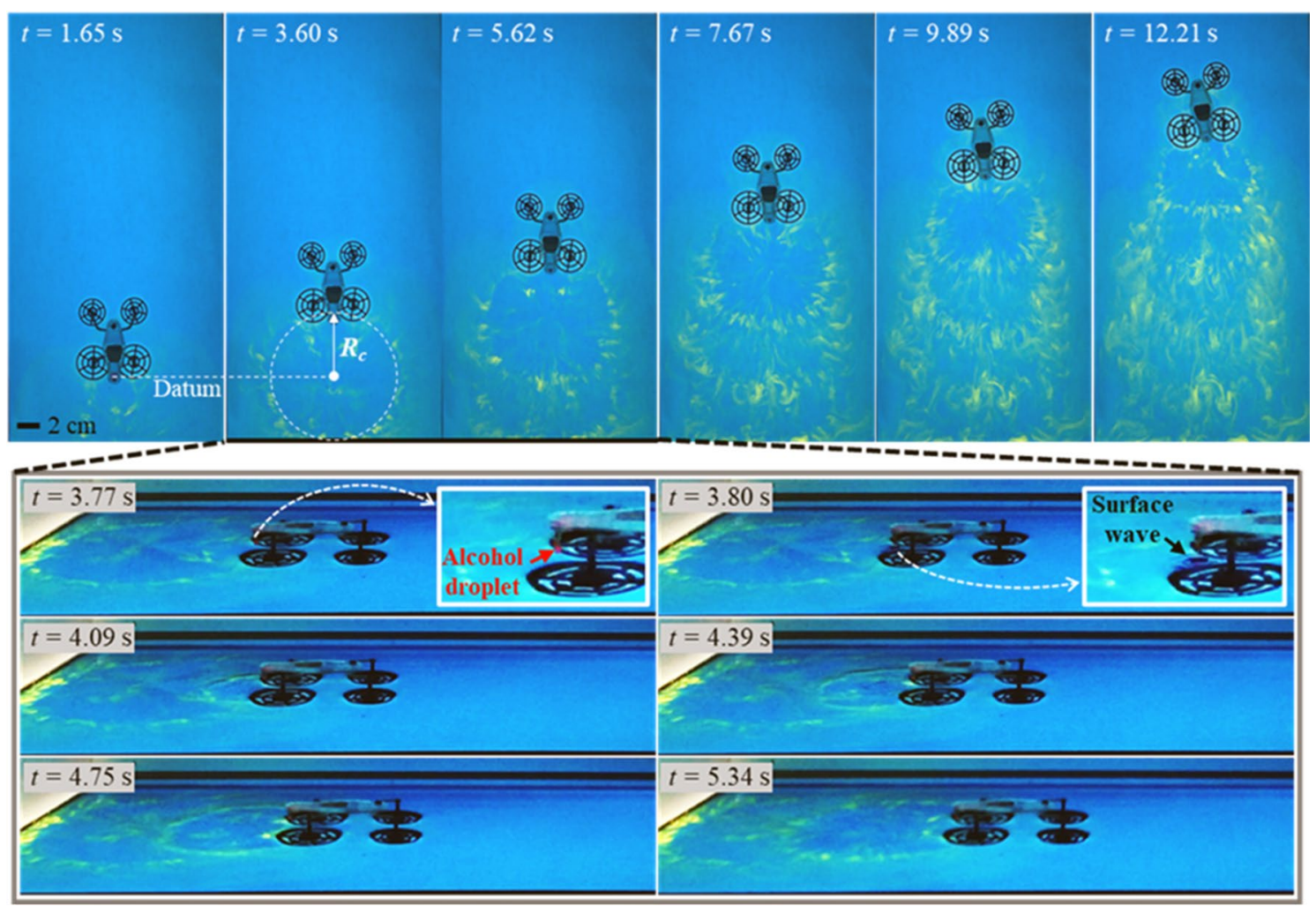

Figure 3. Marangoni flow visualization during propulsion. Top and side views of the robot propelled by the Marangoni effect; flow was visualized using BTB and other chemicals ( $R_{c}$ : ring trace radius). CP served as the porous medium. $D_{O}$ was $3.2 \mathrm{~mm}$ and $h_{f}$ was $9.5 \mathrm{~mm}$; $t$ is the time that elapsed after the first alcohol droplet broke from the nozzle.

respectively. By letting the total volume of injected alcohol as $M_{a}$, the theoretical number of alcohol droplets $\left(N_{\text {drop }}\right)$ is defined in Fig. 2A. In addition, the time between the breakups of two consecutive droplets $\left(T_{\text {drop }}\right)$ is found as $V_{a} / Q_{a}$. Because of the reciprocal term $V_{a}$ between $N_{\text {drop }}$ and $T_{\text {drop }}$, they are inversely related. As it will be shown in locomotion analysis section, both $N_{\text {drop }}$ and $T_{\text {drop }}$ directly affect the locomotion of the robot. Figure $2 \mathrm{C}$ shows the inverse proportional relation of $T_{\text {drop }}$ and $N_{\text {drop }}$ more clearly; note that $N_{\text {drop }}$ was heavily determined by $D_{O}$. Given minor fabrication error and the fact that the liquids were injected manually, slight differences in the $N_{\text {drop }}$ values were observed at the same $D_{O}$. The horizontal lines inside the interquartiles (IQRs) are medians, which were close to the means, and proportional to $D_{O}$ for both FP and CP. The IQRs show that the use of FP and a $D_{O}=3.2 \mathrm{~mm}$ was associated with very variable $T_{\text {drop }}$ values in successive tests, caused by the inherently high variability of paper-based microfluidic devices ${ }^{49}$. Given that the experimental relation between $N_{\text {drop }}$ and $D_{O}$, the theoretical $T_{\text {drop }}$ (i.e. the hollow markers in Fig. 2C) shows good agreement with measured $T_{\text {drop. }}$ However, the theoretical $N_{\text {drop }}$ overestimated a couple of droplets when $D_{O}$ was $2.2 \mathrm{~mm}$ or $3.2 \mathrm{~mm}$ (Supplementary Figure S8) due to the variance of $M_{a}$ during the manual injection.

The time evolution of the droplet counts for each $D_{O}$ are presented in Fig. 2D-G. Here, the time of the first droplet breakup was used as a reference, and the times to later breakups are depicted. CP was associated with faster breakup than FP as expected due to the higher flow rate (see Fig. $2 \mathrm{~B}$ ), and smaller $D_{O}$ values were associated with more droplets of smaller volume. The pump produced a maximum of 13 droplets when $D_{O}=1.0 \mathrm{~mm}$. By considering small mean difference in $T_{\text {drop }}$ values (see Supplementary Note: Pumping periodicity), the pump was also working in reasonably periodic manner.

Marangoni flow visualization during propulsion. To visualize induced Marangoni flow, bromothymol blue (BTB), which changes color depending on $\mathrm{pH}^{50}$, was used as explained in Supplementary Note: Marangoni Flow Visualization. Alcohol spreading behind the moving robot was revealed in yellow (Fig. 3 and Supplementary Video S5). Each image at the top of Fig. 3 was taken when a droplet was fully spread. Interestingly, the ring trace radius $\left(R_{c}\right)$ was almost the same as the travel distance afforded by a single droplet. Also, the center of each ring was the location where the preceding alcohol droplet had coalesced with water.

A side view filmed between 3.77 and 5.34 s shows the radial spreading of a single droplet in more detail, starting with droplet breakup at the nozzle. The radial flow spread was accompanied by many surface waves (see Supplementary Figure S1 1 for closeups) induced by Marangoni effect, confirming that 3Me1Bu facilitated prolonged motion ${ }^{34}$. However, as an additional chemical was used for visualization, the surface tension difference between the water surface and the propellant was only $57 \%$ that of the pure water-and-alcohol case (see 

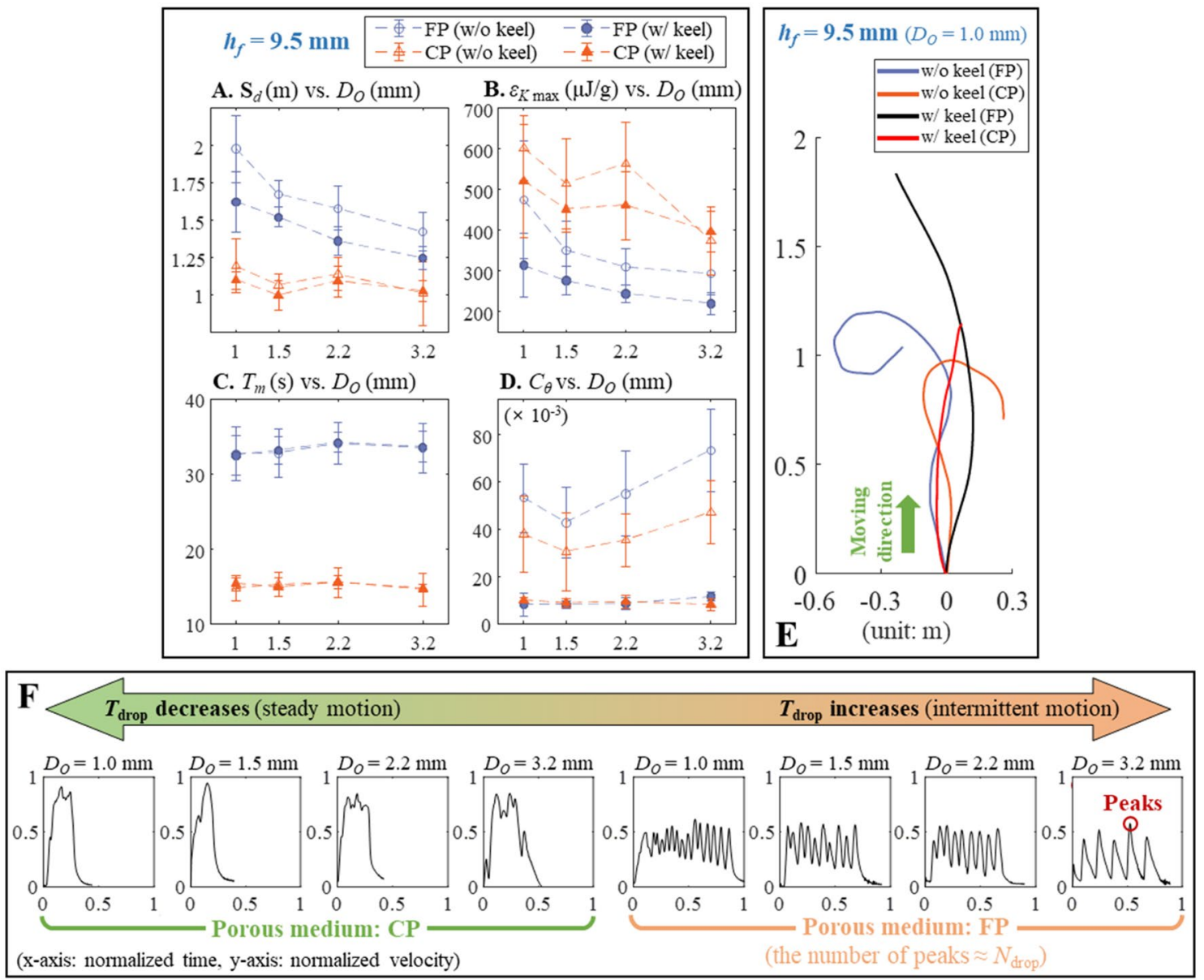

Figure 4. Robot locomotory characteristics at an $h_{f}=9.5 \mathrm{~mm}$. (A) Traveled distance $\left(S_{d}\right)$ versus $D_{O}$. (B) Energy density of fuel $\varepsilon_{K, \max }$ versus $D_{O}$. (C) Duration of motion $T_{m}$ versus $D_{O}$. (D) Moving trajectory characteristic $C_{\theta}$ versus $D_{O}$ ( $n=5$ for $\left.\mathbf{A}-\mathbf{D}\right)$. (E) Moving trajectories of some selected cases. (F) The variation of normalized velocity of the robot upon $T_{\text {drop }}$ change. Note that all the error bars denote standard deviations.

Supplementary Note: Marangoni Flow Visualization). The robot actually traveled less distance than on clear water, but interpretation of the trace left on the water surface yielded information on preceding motion.

Locomotion analysis when $\boldsymbol{h}_{f}=\mathbf{9 . 5} \mathrm{mm}$ : complete droplet breakup. For comprehensive locomotion analysis, we compared traveled distance $S_{d}$, energy density of fuel $\varepsilon_{K \text {,max }}$, duration of motion $T_{m}$, and trajectory characteristic $C_{\theta}$ in Fig. $4 \mathrm{~A}-\mathrm{D}$ by varying the porous media (either $\mathrm{CP}$ or FP), alcohol outlet diameter $D_{O}$, and keel status while holding the foot height $h_{f}$ (Fig. $2 \mathrm{~A}$ ) at $9.5 \mathrm{~mm}$. Note that letting $h_{f}=9.5 \mathrm{~mm}$ ensured complete droplet breakup from the nozzle (see Supplementary Figure S12A,C). Thus, $T_{\text {drop }}$ and $N_{\text {drop }}$ analyzed in Fig. 2 can directly affect the locomotion on water surface in predictable and intuitive manner. Here, $\varepsilon_{K, \max }$ was defined as:

$$
\varepsilon_{K, \max }=\frac{m_{\text {fuel }}}{2 m_{\text {fuel }}} V_{\max }^{2}
$$

where $m_{\text {robot }}$ and $m_{\text {fuel }}$ are the masses of the robot and injected fuel respectively and $V_{\max }$ is the maximum propulsion speed ${ }^{12}$; its unit $[\mu \mathrm{J} / \mathrm{g}]$ indicates that it is equivalent to the maximum kinetic energy per unit mass. In the case of $C_{\theta}$, it is dimensionless and quantitatively characterizes the effect of the keel on directional motion, indicating how the heading angle changes during propulsion. Its definition is given below:

$$
C_{\theta}=\frac{\Delta T\left(\theta_{1}+\theta_{2}+\cdots+\theta_{n-1}\right)}{\pi T_{m}}
$$

where $\Delta T$ is sampling time, and $\theta_{1}+\theta_{2}+\cdots+\theta_{n-1}$ (unit: radian) are series of heading angle changes in $\Delta T$ interval. If the robot proceeded linearly, $C_{\theta}$ was close to zero, and vice versa. Overall, both $S_{d}$ and $\varepsilon_{K, \max }$ were inversely related to $D_{O}$ (Fig. $4 \mathrm{~A}, \mathrm{~B}$ ). The use of FP (compared to $\mathrm{CP}$ ) increased the distance traversed at all $D_{O}$; and CP use was associated with higher $\varepsilon_{K, \max }$ values than FP use. Thus, frequent dispensing of small alcohol droplets (i.e. large $N_{\text {drop }}$ with short $T_{\text {drop }}$ ) was associated with a longer $S_{d}$ and higher $\varepsilon_{K \text {,max }}$ than slow dripping of a few but large droplets. Figure $4 \mathrm{C}$ shows that the $T_{m}$ was predominantly affected by the porous medium chosen; the $T_{m}$ 


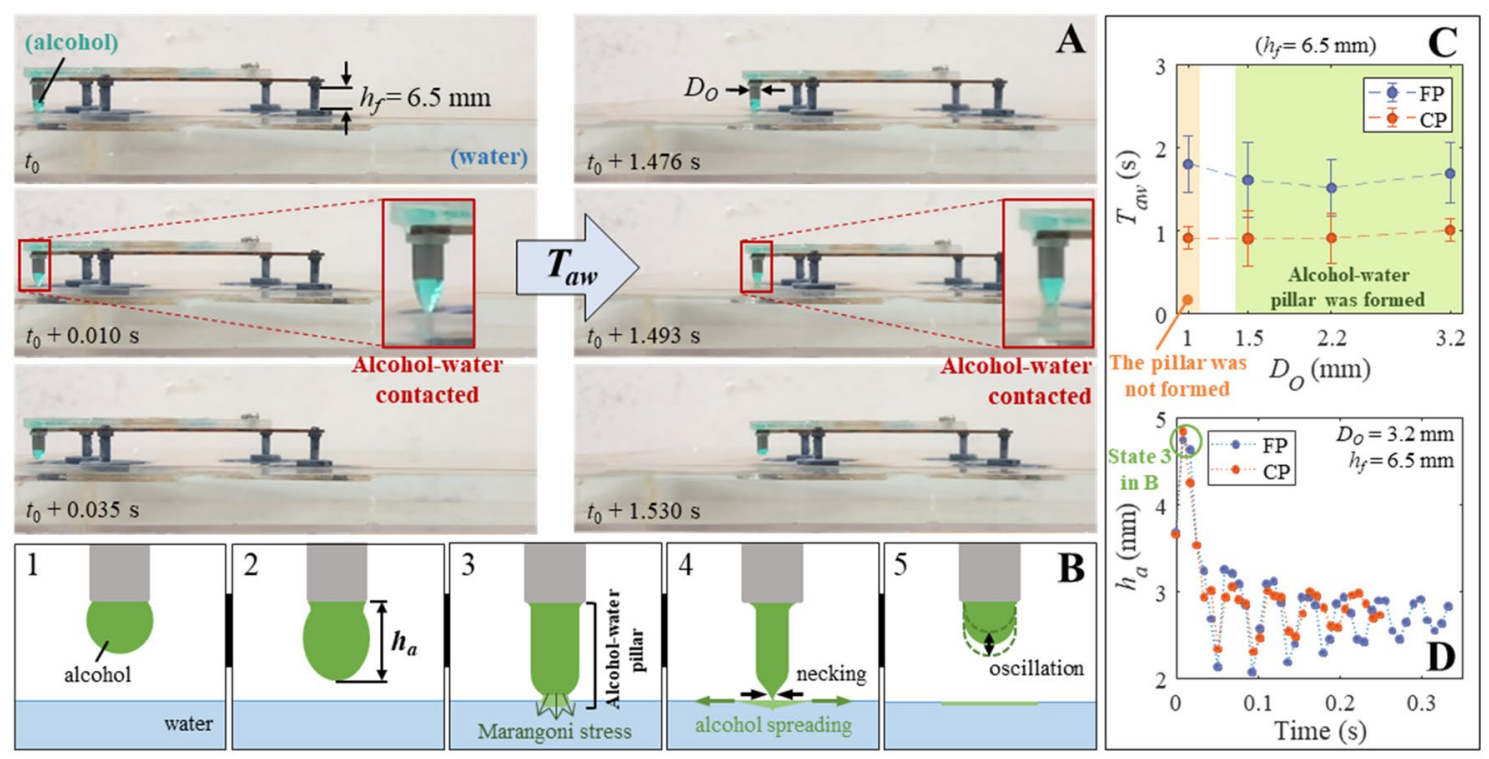

Figure 5. Incomplete droplet breakup at $h_{f}$ of $6.5 \mathrm{~mm}$ forms an alcohol-water pillar. (A) Premature alcoholwater contact at an $h_{f}=6.5 \mathrm{~mm}\left(t_{0}\right.$ : reference time, $T_{a w}$ : time between two episodes of alcohol-water contact). (B) A schematic of alcohol spreading at $h_{f}=6.5 \mathrm{~mm}$. (C) $T_{a w}$ versus $D_{O}(n=5)$. (D) Oscillation of alcohol column height $\left(h_{a}\right)$. Note that all the error bars denote standard deviations.

associated with FP use was always longer than that associated with CP use. Thus, fast water-imbibition into a porous medium (using CP) could boost up $\varepsilon_{K, \max }$ at the expense of short $S_{d}$.

For both porous materials, keel-extrusion reduced both $S_{d}$ and $\varepsilon_{K, \max }$ because of the drag imposed by the keel itself; while the inverse relations with $D_{O}$ were still maintained. However, $C_{\theta}$ obtained from the keel-extrusion was significantly smaller than those of without keels (Fig. 4D). Thus, the keel afforded directional motion as expected and some typical trajectories are depicted in Fig. 4E. Clearly, the red and black curves in Fig. 4E (i.e., smaller $C_{\theta}$ ) were less curvy than the blue and orange curves. This characteristic offered by the keel-extrusion was repeatable judging from the associated small standard deviations in Fig. $4 \mathrm{D}$. On the other hand, $C_{\theta}$ obtained from the keel-free footpads were characterized by large standard deviation, which implied unordered and arbitrarily trajectories. The relation between $T_{\text {drop }}$ and locomotory characteristic is more clearly shown in Fig. $4 \mathrm{~F}$. Each subgraph exhibits the normalized velocity of the robot (without keeled footpads) versus normalized time. Noticeably, using a larger nozzle and FP facilitated the robot to move in intermittent manner. Also, the number of velocity peaks was almost the same as $N_{\mathrm{drop}}$ at the same $D_{O}$; the inversely proportional relation between $D_{O}$ and $N_{\text {drop }}$ in Fig. $2 \mathrm{C}$ was responsible for this tendency. On the other hand, using CP with a smaller nozzle facilitated steady locomotion with less velocity fluctuation. Thus, the number of velocity peaks was not meaningfully distinguishable even though $D_{O}$ was changed. The relation between $T_{\text {drop }}$ and the resultant behavior was still maintained when keel-extruded footpad was employed. As long as a complete droplet breakup is guaranteed, the pump behavior directly affected the locomotion.

Locomotion analysis when $h_{f}=6.5 \mathrm{~mm}$ : incomplete droplet breakup. Reducing $h_{f}=6.5 \mathrm{~mm}$ promoted earlier contact of alcohol to the water surface (Fig. 5A,B) except when $D_{O}=1.0 \mathrm{~mm}$ (see Supplementary Figure S12B,D). Notably, an alcohol-water pillar was momentarily observed (Supplementary Video S6-S7). At the same time, the alcohol spread radially because of the outward Marangoni stress, followed by necking (scenes 3-4 in Fig. 5B $)^{51,52}$. The time between two consecutive alcohol-water contacts $\left(T_{a w}\right)$ was noticeably reduced (Fig. $5 \mathrm{C}$ ), thus to less than the $T_{\text {drop }}$ in Fig. 2C. Finally, the remaining alcohol dangling at the nozzle began to oscillate, especially when $D_{O}=3.2 \mathrm{~mm}$ (Fig. 5D). The impact of this incomplete droplet breakup had affected the locomotion of the robot.

The resultant traveled distance and energy density of fuel exhibited noticeable differences (Fig. 6A,B). Note that it was $D_{O}=1.0 \mathrm{~mm}$ and $D_{O}=3.2 \mathrm{~mm}$ that exhibited maximal $S_{d}$ for $h_{f}=9.5 \mathrm{~mm}$ and $h_{f}=6.5 \mathrm{~mm}$, respectively. This implies that optimal $h_{f}$ for maximal $S_{d}$ would be different if $D_{O}$ is changed. However, smaller $D_{O}$ still facilitated larger $\varepsilon_{K, \max }$ when CP was used. For the time duration and directionality of motion (Fig. 6C,D), the overall tendencies were the same at both $h_{f}$ values. The effect of $h_{f}$ is also evident in the velocity vs. time graphs (Fig. 6E). The velocity of $\left(D_{O}=3.2 \mathrm{~mm}, \mathrm{FP}, h_{f}=6.5 \mathrm{~mm}\right)$ case exhibits more peaks and less fluctuation than that for the $\left(D_{O}=3.2 \mathrm{~mm}, \mathrm{FP}, h_{f}=9.5 \mathrm{~mm}\right)$ case, given the more frequent alcohol-water contacts of the former case. When $h_{f}=9.5 \mathrm{~mm}$, the velocity increased once more after becoming near zero. Given such rises followed by gradual decreases, the resultant $S_{d}$ at $h_{f}=9.5 \mathrm{~mm}$ (Fig. $4 \mathrm{~A}$ ) was comparatively small. The impact of $T_{a w}$ to the velocity of the robot is summarized in Fig. 6F. Using CP again facilitated steady motion, while FP induced noticeable velocity fluctuations. Unlike Fig. 4F, however, the number of velocity peaks obtained from FP were hardly correlated with $D_{O}$. As $D_{O}$ had less effect to $T_{a w}$ (Fig. $5 \mathrm{C}$ ), significant change in the velocity profile was only induced by porous media. This tendency was maintained when the keel was present except the enhanced 

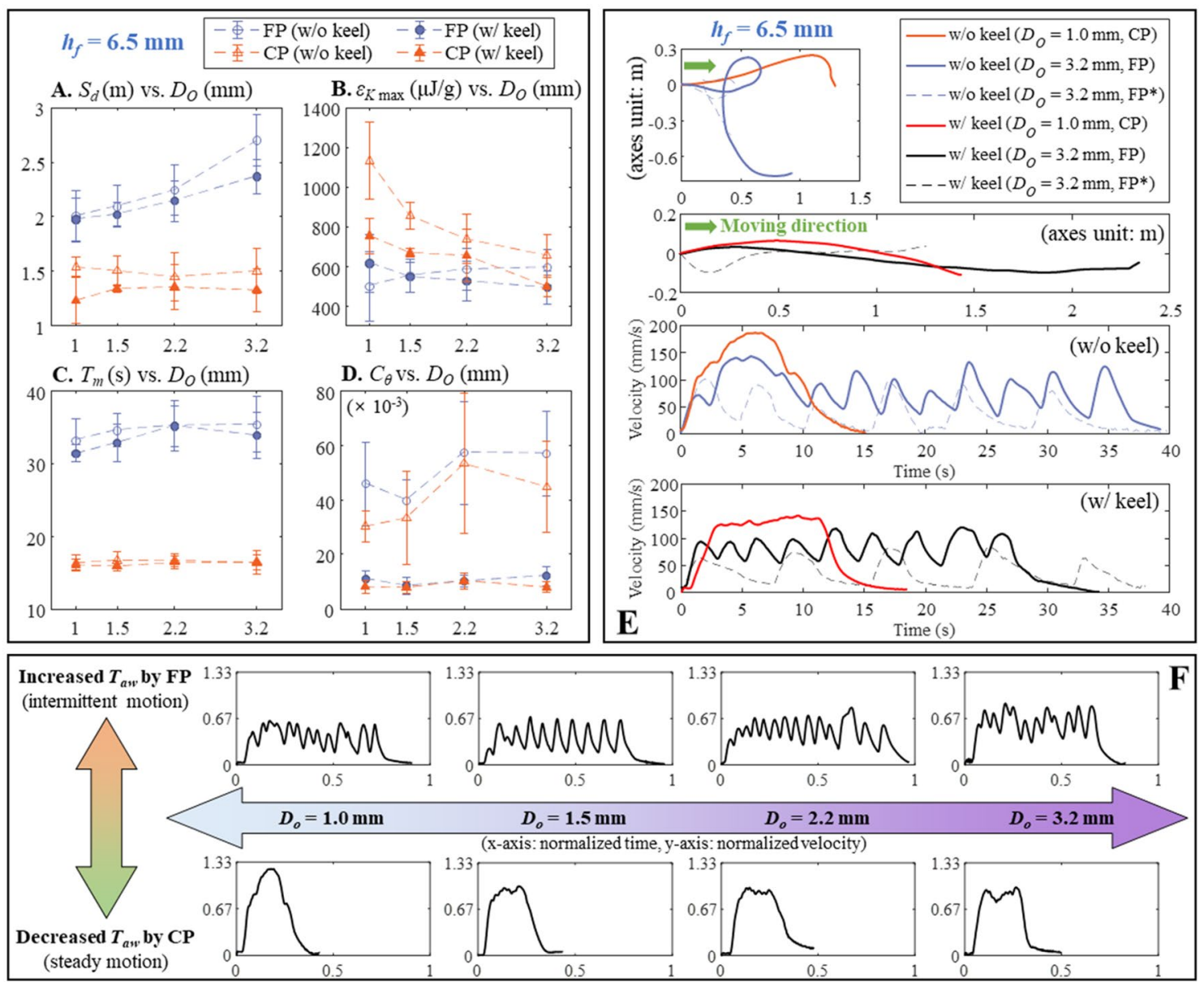

Figure 6. Robot locomotory characteristic at $h_{f}=6.5 \mathrm{~mm}$. (A) Traveled distance $\left(S_{d}\right)$ versus $D_{O}$. (B) Energy density of fuel $\varepsilon_{K, \max }$ versus $D_{O}$. (C) Duration of motion $T_{m}$ versus $D_{O}$. (D) Moving trajectory characteristic $C_{\theta}$ versus $D_{O}$ ( $n=5$ for $\left.\mathbf{A}-\mathbf{D}\right)$. (E) Variation of velocity and corresponding moving trajectories of some selected cases. (F) The variation of normalized velocity of the robot upon $T_{a w}$ change; note that Fig. $4 \mathrm{~F}$ and (F) were normalized in the same scale. Note that all the error bars denote standard deviations.

directional motion at the expense of velocity. Essentially, the time interval of repetitive alcohol-water contacts and the amounts of alcohol discharged for each contact determine the locomotory behavior. When $h_{f}=6.5 \mathrm{~mm}$, smaller $T_{a w}$ than $T_{\text {drop }}$ had enhanced both $S_{d}$ and $\varepsilon_{K, \max }$. This implies that premature contact between (incompletely grown) alcohol and water surface, and underlying dynamics during the alcohol-to-water coalescence (Fig. 5B) are key aspects for efficient locomotion.

The relations between target performance and design parameters. Each sets of design parameters were specialized at achieving different locomotory performance (refer Supplementary Video S8). To travel the longest distance, using FP and keel-free-footpad is required. At the same time, nozzle diameter should be small for complete droplet breakup $\left(D_{O}=1.0 \mathrm{~mm}, h_{f}=9.5 \mathrm{~mm}\right)$, and large for incomplete droplet breakup $\left(D_{O}=3.2 \mathrm{~mm}, h_{f}=6.5 \mathrm{~mm}\right)$. To achieve the fast speed, using CP, keel-free-footpad, and small nozzle are required. To maintain directional motion, using a keel-extruded footpad is sufficient. Whereas using FP is sufficient for prolonged operation of the robot independent to other design parameters. In terms of velocity variation, using $\mathrm{CP}$ is recommended for steady locomotion. If possible, using small $D_{O}$ or letting $h_{f}=6.5 \mathrm{~mm}$ facilitates better steady motion. On the other hand, FP is applicable for intermittent locomotion. If possible, using larger $D_{O}$ or letting $h_{f}=9.5 \mathrm{~mm}$ is recommended.

Locomotory characteristics of the proposed robot and others. To further compare the locomotory characteristics with other systems and some aquatic arthropods, $\varepsilon_{K, \max }$, maximum Weber number $\left(W e_{\max }\right)$, and maximum Reynolds number $\left(R e_{\max }\right)$ were considered. Here, $W e_{\max }$ and $R e_{\max }$ were dimensionless and defined as below:

$$
W e_{\max }=\frac{\rho_{w} w_{f} V_{\max }^{2}}{\sigma_{w}}, \quad R e_{\max }=\frac{V_{\max } w_{f}}{v_{w}}
$$




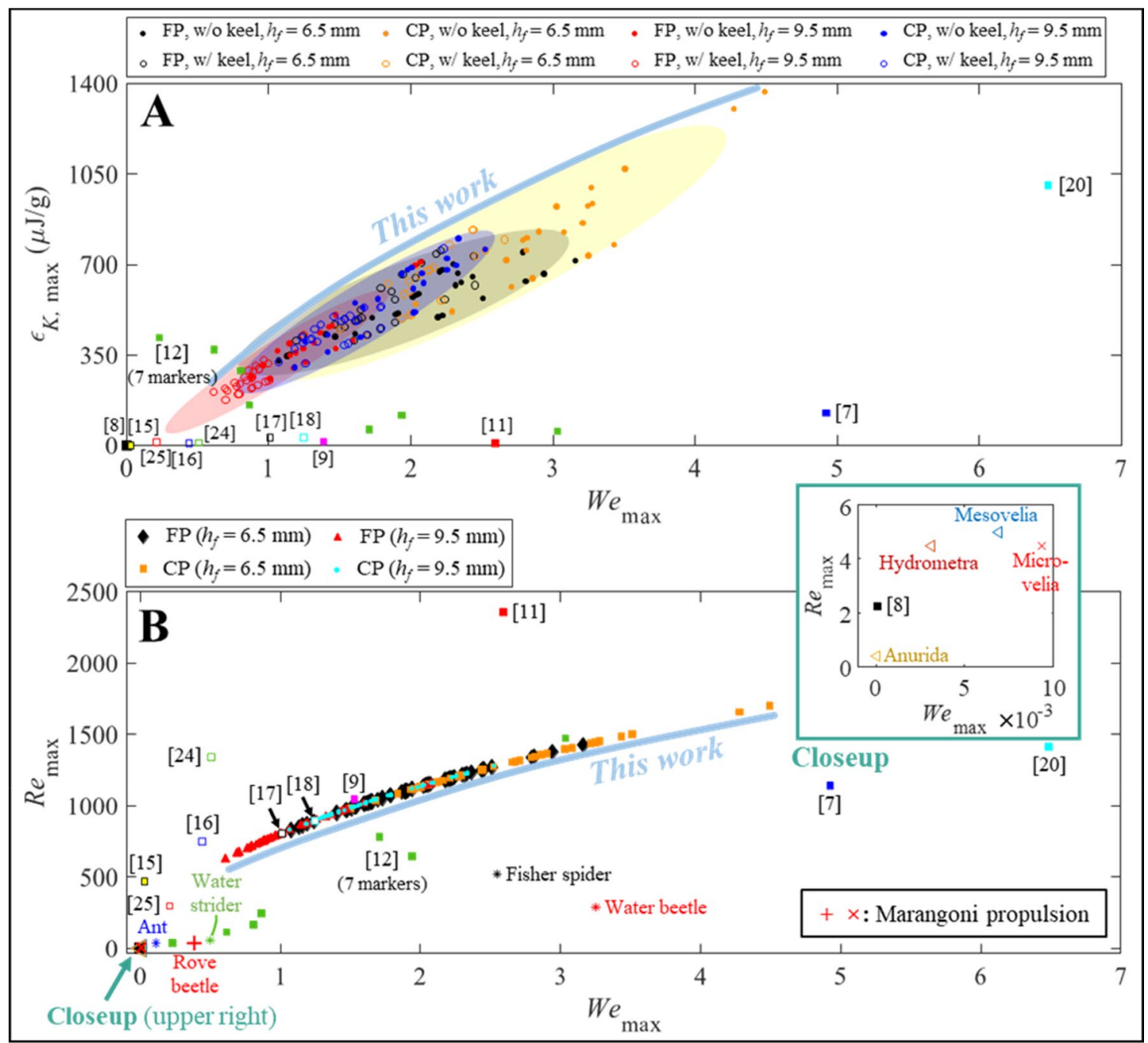

Figure 7. Robot locomotory characteristic using nondimensional numbers. (A) $\varepsilon_{K, \max }$ versus $W e_{\max }$. Error eclipses include $95 \%$ confidence intervals. Identical coloration of markers and eclipses indicate the use of identical porous media and $h_{f}$ values. (B) $R e_{\max }$ versus $W e_{\max }$. Biological data were obtained from ${ }^{2,53-57}$, and no distinguish was made between keeled and pristine footpads.

where $w_{f}$ was foot width, and $v_{w}$ was kinematic viscosity of water ${ }^{1} . W e_{\max }$ and $R e_{\max }$ indicate the importance of inertial force compared to surface tension and viscous force, respectively. Depending on $V_{\max }$ of the robot, its $W e_{\max }$ was varied from 0.5 to 4.5 (Fig. 7), thus within the ranges of many previous works. In particular, the $\varepsilon_{K, \max }$ of the (CP, w/o keel, $\left.h_{f}=6.5 \mathrm{~mm}\right)$ cases were higher than those in any previous works; the highest $\varepsilon_{K \text {,max }}$ in our work was $1368 \mu \mathrm{J} / \mathrm{g}$. Note that specific parameters used to depict Fig. 7 are summarized in Supplementary Table S3. In the case of $R e_{\max }$, its range was also similar to that of previous systems. However, $R e_{\max }$ of this work was far higher than that of aquatic arthropods. Here, only the red cross and plus markers denoted data originated from Marangoni propulsion; while other markers were obtained from other modes of locomotion for comparison ${ }^{2,53-57}$. This implies that the robot behavior was dominated by inertia due to its larger body size than arthropods.

\section{Discussion}

Capillarity is one of the fundamental physical phenomena that enables autonomous fluid flow without an external assistance. This study demonstrates how capillary pressure can provide mobility to a robot on water surface. Inspired by the Marangoni propulsion of aquatic insects, a new battery-less self-propulsion robot was developed, which utilized a magnetically triggerable, water-imbibition powered microfluidic pump for the first time. Owing to utilization of Marangoni effect, no electrically driven actuator, or other forms of mechanically moveable parts are required to generate thrust. While previous Marangoni propulsion systems had difficulties in controlling the propellant-transfer to water surface, the dripping behaviors of propellant are mechanically tunable in this work. Our method no longer required a vertically-aligned vessel to generate fuel droplets unlike previous systems ${ }^{15-18}$. Thus, the proposed system could avoid any accompanying issue such as the submergence caused by the unequally distributed load from a fully loaded fuel vessel. The trigger mechanism of the pump employed 
a novel microfluidic structure, which was not tried in the previous methods ${ }^{16-18,23,27}$. The proposed trigger is electric power free, instantaneous, independent to water surface condition, and free from an external equipment. The pump performance was also comprehensively analyzed. It was shown that the locomotion of the robot can be related with the predicted pump characteristic if the complete droplet breakup is ensured. On the other hand, approaching the nozzle tip closer to water surface increased the complexity of the locomotory behavior. More specifically, the locomotory characteristics were significantly different depending on four parameters: namely, porous materials (FP or CP), keel-extrusion, nozzle diameter $\left(D_{O}\right)$, and footpad height $\left(h_{f}=6.5\right.$ or $\left.9.5 \mathrm{~mm}\right)$. Overall, using CP was characterized by fast propulsion speed in steady manner but with short duration because of the fast water-imbibition rate than FP (refer Fig. 2). Adsorbing the same amount of water with a fast flow rate inevitably reduced the operational time of the pump. Thus, using FP was associated with prolonged propulsion than CP because of its smaller flow rate than CP. In the case of keel-extrusion, it certainly helped the robot to maintain directional motion independent to other parameters (refer Figs. 4D, 6D). This was possible due to the asymmetric drag force generated by the keel, which already explained in Supplementary Note: Drag asymmetry. Whereas the effects of $D_{O}$ and $h_{f}$ were tightly coupled as a small $h_{f}$ facilitated premature contact of a propellant droplet to the water surface. When $h_{f}=9.5 \mathrm{~mm}$ (i.e., complete droplet breakup), increasing $D_{O}$ reduced both the traveled distance $\left(S_{d}\right)$ and maximal propulsion speed (or $\varepsilon_{K, \max }$ ) due to the relatively long $T_{\text {drop }}$ at large $D_{O}$ (refer Fig. 2C). An overly long $T_{\text {drop }}$ could not provide a propulsive force while the robot was still propelled by a prior propellant. Intermittent propulsion after when the velocity of the robot was reduced nearly zero had a disadvantage in achieving a longer traveled distance with fast propulsion speed. On the other hand, reducing $h_{f}$ down to $6.5 \mathrm{~mm}$ complicated all the kinematic result as reported in Fig. 6. But, both $S_{d}$ and $\varepsilon_{K, \max }$ was improved owing to the frequent dispensing of propellant (refer Fig. 5C) than the dispensing rate associate with $h_{f}=9.5 \mathrm{~mm}$ (i.e. $T_{\text {drop }}$ in Fig. $2 \mathrm{C}$ ).

Changing the porous material effectively changed the flow rate of the pump, and similar result can also be obtained by varying the microchannel-porous medium heights $(H)$. As already addressed in equations $\mathrm{S} 10$ and S16 (Supplementary Note: Flow rate model), $H$ was proportional to the flow rate. Thus, increasing $H$ would have similar results of switching the porous medium from FP to CP: reduced $T_{\text {drop }}, S_{d}$, and $T_{m}$, but increased $\varepsilon_{K \max }$. On the other hand, decreasing of $H$ will exhibit the opposite locomotory behaviors.

The proposed system, however, exhibited noticeable performance-variability caused by multi-layered filter papers and manually packed cellulose powders. Thus, a tailored porous medium should be fabricated in desired size and shape for consistent performance. Also, the density of microchannel should be increased (to hold more fuel) for a given volume of the pump to increase the limited operation time. In addition, measuring and modeling of the time-varying Marangoni propulsion force in our system is still remained as a great challenge ${ }^{58}$. This work is still a foundational study, which integrated a microfluidic device to a liquid interface traveling system, and future investigation is required for its potential applications. For example, the proposed system can be used to environmental remediation of liquid interface ${ }^{22}$. Besides, the functionality of on-board microfluidic device can be improved by integrating microfluidic digital $\operatorname{logic} c^{59}$, or a passive microfluidic mixer ${ }^{60}$ to induce on-site chemical reactions.

\section{Methods}

Preparation of porous media. Off-the-shelf filter papers (see Supplementary Table S1) and cellulose powder (Daejung Chemicals) were used. Filter papers were cut with a $\mathrm{CO}_{2}$ laser machine. The particle retention and basis weight specified in Supplementary Table S1 were obtained from the manufactures' datasheet. While the total thickness was measured using a digital caliper in $0.01 \mathrm{~mm}$ resolution. The particle size distribution of the cellulose powder was obtained from a particle size analyzer (Mastersizer 3000. Malvern).

Fabrication of the proposed robot. An exploded view of the robot is given in Supplementary Figure S13. The pump was composed of two layers made of polydimethylsiloxane elastomer (Sylgard 184, Dow-corning), and both layers were molded and cured in an oven for $2 \mathrm{~h}$ at $60^{\circ} \mathrm{C}$ after pouring liquid state elastomer into 3D printed molds. These two layers were bonded each other using a corona plasma treater (Corona SB, BlackHole Lab) followed by bonding a neodymium magnet to the bottom layer with an adhesive (Loctite 401, Henkel). A SUS nozzle was also tightly fitted into the outlet. After that, a laser-cut $0.8 \mathrm{~mm}$ thick polycarbonate body frame was bonded with the pump using an adhesive layer (468MP, $3 \mathrm{M}$ ). The circular footpads were made of 3D printed acrylonitrile butadiene styrene. Whereas a $0.15 \mathrm{~mm}$ thick epoxy-impregnated glass fiber plate was for the keel. The footpads were all spray coated with water-repelling material (NeverWet, RUST-OLEUM) for hydrophobicity. Before injecting water and alcohol to the microchannels, a porous medium is filled inside the designated chamber. The porous medium was gently pressed with a laser-cut polycarbonate cover followed by sealing with an adhesive tape for airtightness.

Measurement of capillary parameters. To theoretically calculate the flow rate of water-imbibition, capillary pressure $\left(P_{c}\right)$, porosity $(\phi)$, contact angle $(\theta)$ between water and porous medium, and mean pore radius $\left(r_{m}\right)$ were required. Here, $P_{c}$ was obtained by measuring the maximum blockage pressure at the terminal of a porous medium while water was being imbibed into it (Supplementary Note: Capillary pressure measurement). The porosities of filter papers and cellulose powder were found by using mercury porosimetry (Supplementary Note: Porosity measurement). Lastly, $\theta$ and $r_{m}$ were found according to the previous method $^{61}$; they were parameterized to a nonlinear fitting problem of water adsorption mass versus time (Supplementary Note: Contact angle and mean pore radius). 
Filming and measurement of dripping interval, flow rates, and alcohol droplets. All the time intervals in Fig. 2 were obtained by filming the outlet nozzle at $30 \mathrm{fps}$ (frame-per-second) and inspecting the moment of droplet breakup frame-by-frame. Here, the pump was fixed to a stationary platform, and each case was repeated 15 times. To measure the flow rates in Supplementary Figure S6A, a transparent film printed with calibrations along the microchannel was aligned on the pump. Then, the entire pump operation was filmed (FP: $60 \mathrm{fps}, \mathrm{CP}: 120 \mathrm{fps})$. We also employed another camera directing to the outlet nozzle to identify the moments of droplet breakup. Flow rate was calculated by multiplying the cross-section area of the microchannel $\left(0.75 \mathrm{~mm}^{2}\right)$ with the speed of water or alcohol moving inside. As we already knew all the distances between two calibrations, the moving speed of the liquids for each unit distance could be easily known. The average flow rates in Fig. 2B and Supplementary Figure S6B were obtained from the series of flow rate vs. time graphs. The alcohol-water pillars shown in Fig. 5A were recorded by filming the robot moving on a water filled petri dish (size: $24 \times 24 \times 2.5$ $\mathrm{cm}^{3}$ ) at $120 \mathrm{fps}$. After that, $T_{a w}$ was obtained by measuring the time interval of the contact between alcohol and water had sequentially occurred. In the case of Supplementary Figure S12, the same water-filled petri dish was used; however, the robot was set to immovable (by placing heavy weights) to get clear images $\left(h_{f}=9.5 \mathrm{~mm}: 60\right.$ fps, $h_{f}=6.5 \mathrm{~mm}\left[D_{O}=2.2,3.2 \mathrm{~mm}, \mathrm{FP}\right]: 60 \mathrm{fps}, h_{f}=6.5 \mathrm{~mm}\left[D_{O}=1.0,1.5 \mathrm{~mm}\right]: 240 \mathrm{fps}$, otherwise: $\left.120 \mathrm{fps}\right)$. The oscillation of alcohol height $h_{a}$ was measured by using ImageJ.

Marangoni flow visualization. To demonstrate intuitive Marangoni flow visualization during the propulsion of the robot, bromothymol blue (BTB) was utilized, which was changed into yellow and blue color when $\mathrm{pH}$ was acidic and alkaline, respectively. Thus, we adjusted the $\mathrm{pH}$ of water inside a $25 \times 75 \mathrm{~cm}^{2}$ sized water tank to 7.6 (weak alkaline); while the $\mathrm{pH}$ of alcohol fuel was set to 1.3 (strong acidic). More detail procedures were fully described in Supplementary Note: Marangoni Flow Visualization.

Force measurement of the footpads. Maximal support force was measured by monitoring the sudden drop of reaction force while a footpad was slowly approaching toward water surface until the integrity of water surface was broken (Supplementary Note: Maximal supporting force). Whereas, dragging a keel-extruded footpad at constant speed inside a water tank could calculate the drag asymmetry in parallel flow direction and normal direction (Supplementary Note: Drag asymmetry).

Motion measurement on water surface. The locomotion of the robot was measured with eleven motion capture cameras (Prime 13, Optitrack) placed around a $3.5 \times 2.1 \mathrm{~m}^{2}$ sized water pool. A marker was attached to the robot for tracking its moving trajectory at $120 \mathrm{~Hz}$. If certain data was partly lost during the measurement, cubic spline was performed to fill the gap. Also, a low pass filter, which was pre-implemented in Motive software (Optitrack), was applied to the position data at $4 \mathrm{~Hz}$ of cut-off frequency to remove spikes. The instantaneous velocity of the robot was obtained by dividing $1 / 120 \mathrm{~s}$ (i.e. the inverse of data acquisition rate) from the moving distance. This was then post-processed with butterworth filter at $3 \mathrm{~Hz}$ cut-off frequency using Matlab (Mathworks). All the locomotion analysis in Figs. 4, 6, and 7 were acquired in the same manner and repeated 5 times.

Received: 9 July 2021; Accepted: 11 August 2021

Published online: 01 September 2021

\section{References}

1. Bush, J. W. M. \& Hu, D. L. Walking on water: Biolocomotion at the interface. Annu. Rev. Fluid Mech. 38, 339-369 (2006).

2. Bush, J. W. M., Hu, D. L. \& Prakash, M. The integument of water-walking arthropods: Form and function. Adv. Insect Physiol. 34, 117-192 (2008).

3. Nachtigall, W. Locomotion: Mechanics and hydrodynamics of swimming in aquatic insects. In The Physiology of Insecta (ed. Rockstein, M.) 381-432 (Academic Press, 1974).

4. Scriven, L. E. \& Sternling, C. V. The marangoni effects. Nature 187, 186-188 (1960).

5. Kwak, B. \& Bae, J. Locomotion of arthropods in aquatic environment and their applications in robotics. Bioinspir. Biomim. 13, 041002 (2018).

6. Pimienta, V. \& Antoine, C. Self-propulsion on liquid surfaces. Curr. Opin. Colloid Interface Sci. 19, 290-299 (2014).

7. Bassik, N., Abebe, B. T. \& Gracias, D. H. Solvent driven motion of lithographically fabricated gels. Langmuir 24, 12158-12163 (2008).

8. Sharma, R., Chang, S. T. \& Velev, O. D. Gel-based self-propelling particles get programmed to dance. Langmuir 28, 10128-10135 (2012).

9. Matsuda, Y., Suematsu, N. J., Kitahata, H., Ikura, Y. S. \& Nakata, S. Acceleration or deceleration of self-motion by the Marangoni effect. Chem. Phys. Lett. 654, 92-96 (2016).

10. Cheng, M., Zhang, D., Zhang, S., Wang, Z. \& Shi, F. Tackling the short-lived marangoni motion using a supramolecular strategy. CCS Chem. 1, 148-155 (2019).

11. Zhang, L. et al. Marangoni effect-driven motion of miniature robots and generation of electricity on water. Langmuir 33, 1260912615 (2017).

12. Pena-Francesch, A., Giltinan, J. \& Sitti, M. Multifunctional and biodegradable self-propelled protein motors. Nat. Commun. 10, 3188 (2019).

13. Nakata, S. et al. Self-rotation of a camphor scraping on water: New insight into the old problem. Langmuir 13, 4454-4458 (1997).

14. Nakata, S. \& Arima, Y. Self-motion of a phenanthroline disk on divalent metal ion aqueous solutions coupled with complex formation. Colloids Surf. A Physicochem. Eng. Asp. 324, 222-227 (2008).

15. Su, M. Liquid mixing driven motions of floating macroscopic objects. Appl. Phys. Lett. 90, 144102 (2007).

16. Kwak, B. \& Bae, J. Skimming and steering of a non-tethered miniature robot on the water surface using marangoni propulsion. In Proceedings of IEEE/RSJ Int. Conf. on Intelligent Robots and Systems, 3217-3222 (2017). 
17. Kwak, B., Lee, D. \& Bae, J. Flexural joints for improved linear motion of a marangoni propulsion robot: Design and experiment. In Proceedings of IEEE Int. Conf. on Biomedical Robotics and Biomechatronics, 1321-1326 (2018).

18. Kwak, B., Choi, S. \& Bae, J. Directional motion on water surface with keel extruded footpads propelled by Marangoni effect. IEEE Robot. Autom. Lett. 5, 6829-6836 (2020).

19. Burton, L. J., Cheng, N. \& Bush, J. W. M. The cocktail boat. Integr. Comp. Biol. 54, 969-973 (2014).

20. Luo, C., Li, H., Qiao, L. \& Liu, X. Development of surface tension-driven microboats and microflotillas. Microsyst. Technol. 18, 1525-1541 (2012).

21. Lyu, L. X. et al. Bio-inspired untethered fully soft robots in liquid actuated by induced energy gradients. Nat. Sci. Rev. 6, 970-981 (2019).

22. Kong, L., Ambrosi, A., Nasir, M. Z. M., Guan, J. \& Pumera, M. Self-propelled 3D-printed “aircraft carrier" of light-powered smart micromachines for large-volume nitroaromatic explosives removal. Adv. Funct. Mater. 29, 1903872 (2019).

23. Xiao, M., Cheng, M., Zhang, Y. \& Shi, F. Combining the Marangoni effect and the $\mathrm{pH}$-responsive superhydrophobicity-superhydrophilicity transition to biomimic the locomotion process of the beetles of genus Stenus. Small 9, 2509-2514 (2013).

24. Jin, H., Marmur, A., Ikkala, O. \& Ras, R. H. A. Vapour-driven Marangoni propulsion: Continuous, prolonged and tunable motion. Chem. Sci. 3, 2526-2529 (2012).

25. Musin, A., Grynyov, R., Frenkel, M. \& Bormashenko, E. Self-propulsion of a metallic superoleophobic micro-boat. J. Colloid Interface Sci. 479, 182-188 (2016).

26. Zhang, H., Duan, W., Liu, L. \& Sen, A. Depolymerization-powered autonomous motors using biocompatible fuel. J. Am. Chem. Soc. 135, 15734-15737 (2013).

27. Chu, Y., Qin, L., Zhen, L. \& Pan, Q. Controlled movement of a smart miniature submarine at various interfaces. ACS Appl. Mater. Interfaces. 10, 24899-24904 (2018).

28. Okabe, S., Sakamoto, K., Murakami, Y., Ishikawa, T. \& Miyake, R. Micro-liquid handling on paper analysis chip triggered by ink-jet droplet. In Proceedings of 16th International Conference on Solid-State Sensors, Actuators and Microsystems, 318-321 (2011).

29. Wang, X., Hagen, J. A. \& Papautsky, I. Paper pump for passive and programmable transport. Biomicrofluidics 7, 014107 (2013).

30. Dosso, F. D., Kokalj, T., Belotserkovsky, J., Spasic, D. \& Lammertyn, J. Self-powered infusion microfluidic pump for ex vivo drug delivery. Biomed. Microdevices. 20, 44 (2018).

31. Wehner, M. et al. An integrated design and fabrication strategy for entirely soft, autonomous robots. Nature 536, 451-455 (2016).

32. Rothemund, P. et al. A soft, bistable valve for autonomous control of soft actuators. Sci. Robot. 3, eaar7986 (2018).

33. Ozcan, O., Wang, H., Taylor, J. D. \& Sitti, M. STRIDE II: A water strider-inspired miniature robot with circular footpads. Int. J. Adv. Robot. Syst. 11, 1-11 (2014).

34. Renney, C., Brewer, A. \& Mooibroek, T. J. Easy demonstration of the Marangoni effect by prolonged and directional motion: "Soap boat 2.0". J. Chem. Educ. 90, 1353-1357 (2013).

35. Shou, D. et al. Geometry-induced asymmetric capillary flow. Langmuir 30, 5448-5454 (2014).

36. Fu, E., Ramsey, S. A., Kauffman, P., Lutz, B. \& Yager, P. Transport in two-dimensional paper networks. Microfluid. Nanofluidics. 10, 29-35 (2011).

37. Mendez, S. et al. Imbibition in porous membranes of complex shape: Quasi-stationary flow in thin rectangular segments. Langmuir 26, 1380-1385 (2010).

38. Che, Z., Wong, T. N., Nguyen, N.-T., Yap, Y. F. \& Chai, J. C. Numerical investigation of upstream pressure fluctuation during growth and breakup of pendant drops. Chem. Eng. Sci. 66, 5293-5300 (2011).

39. Jackson, G. W. \& James, D. F. The permeability of fibrous porous media. Can. J. Chem. Eng. 64, 364-374 (1986).

40. Kruczek, B. Carman-Kozeny equation. In Encyclopedia of Membranes (eds Drioli, E. \& Giorno, L.) 1-3 (Springer, 2014).

41. Scheidegger, A. E. The Physics of Flow Through Porous Media (University of Toronto Press, 1974)

42. Nield, D. A. \& Bejan, A. Convection in Porous Media (Springer, 2013).

43. Hyväluoma, J. et al. Simulation of liquid penetration in paper. Phys. Rev. E. 73, 036705 (2006).

44. Conrath, M., Fries, N., Zhang, M. \& Dreyer, M. E. Radial capillary transport from an infinite reservoir. Transp. Porous Media. 84, 109-132 (2010).

45. Elizalde, E., Urteaga, R. \& Berli, C. L. A. Rational design of capillary-driven flows for paper-based microfluidics. Lab. Chip. 15, 2173-2180 (2015).

46. Benner, E. M. \& Petsev, D. N. Potential flow in the presence of a sudden expansion: Application to capillary driven transport in porous media. Phys. Rev. E. 87, 033008 (2013).

47. Zhang, X. \& Basaran, O. A. An experimental study of dynamics of drop formation. Phys. Fluids 7, 1184-1203 (1995).

48. Zhang, Z.-Q. \& Mori, Y. H. Formulation of the Harkins-Brown correction factor for drop-volume description. Ind. Eng. Chem. Res. 32, 2950-2952 (1993).

49. Walji, N. \& MacDonald, B. D. Influence of geometry and surrounding conditions on fluid flow in paper-based devices. Micromachines. 7, 73 (2016).

50. Harris, D. C. \& Lucy, C. A. Quantitative Chemical Analysis (W. H. Freeman, 2020).

51. Haldar, K., Chakraborty, S. \& Chakraborty, S. Influence of Marangoni stress on the variation in number of coalescence cascade stages. Can. J. Chem. Eng. 97, 983-994 (2019).

52. Blanchette, F., Messio, L. \& Bush, J. W. M. The influence of surface tension gradients on drop coalescence. Phys. Fluids. 21, 072107 (2009).

53. Hu, D. L. \& Bush, J. W. M. The hydrodynamics of water-walking arthropods. J. Fluid Mech. 644, 5-33 (2010).

54. Hu, D. L. The Hydrodynamics of Water-walking Insects and Spiders, Doctoral dissertation, Massachusetts Institute of Technology, Cambridge, MA (2006).

55. Xu, Z., Lenaghan, S. C., Reese, B. E., Jia, X. \& Zhang, M. Experimental studies and dynamics modeling analysis of the swimming and diving of whirligig beetles (Coleoptera: Gyrinidae). PLoS Comput. Biol. 8, e1002792 (2012).

56. Betz, O. Performance and adaptive value of tarsal morphology in rove beetles of the genus Stenus (Coleoptera, Staphylinidae). J. Exp. Biol. 205, 1097-1113 (2002).

57. Schildknecht, H. Chemical ecology-A chapter of modern natural products chemistry. Angew. Chem. Int. Ed. Engl. 15, 214-222 (1976).

58. Lauga, E. \& Davis, A. M. J. Viscous Marangoni propulsion. J. Fluid Mech. 705, 120-133 (2012).

59. Zhang, Q. et al. Logic digital fluidic in miniaturized functional devices: Perspective to the next generation of microfluidic lab-onchips. Electrophoresis 38, 953-976 (2017).

60. Lee, C.-Y., Wang, W.-T., Liu, C.-C. \& Fu, L.-M. Passive mixers in microfluidic systems: A review. Chem. Eng. J. 288, 146-160 (2016).

61. Demlehner, U. The contact angle of liquids in porous media. Stud. Surf. Sci. Catal. 62, 97-104 (1991).

\section{Acknowledgements}

This work was supported in part by the National Research Foundation of Korea and in part by the Korean Government (MSIT) (No. NRF-2019R1A2C2084677, No. NRF-2016R1A5A1938472). 


\section{Author contributions}

B.K., and J.B. designed the study. B.K., S.C., and J.M fabricated the robot and samples for experiments. B.K., and S. C. performed experiments and analyzed the data of alcohol dripping. B.K. measured the flow rates and designed the theoretical models for the water imbibition. S.C., and J.M. visualized and filmed the Marangoni flow. B.K., S.C., and J.M measured the locomotion of the robot. B.K., and J.B. analyzed the locomotion data. B.K. analyzed the characteristics of the footpad. B.K. measured the successful rate of the magnetic clamping and gauge pressure during the imbibition. J.M. measured the contact angle and mean pore radius of porous media. S.C. filmed the formation of alcohol-water pillar. All authors contributed to the writing of the manuscript.

\section{Competing interests}

The authors declare no competing interests.

Additional information

Supplementary Information The online version contains supplementary material available at https://doi.org/ 10.1038/s41598-021-96553-8.

Correspondence and requests for materials should be addressed to J.B.

Reprints and permissions information is available at www.nature.com/reprints.

Publisher's note Springer Nature remains neutral with regard to jurisdictional claims in published maps and institutional affiliations.

(c) (i) Open Access This article is licensed under a Creative Commons Attribution 4.0 International License, which permits use, sharing, adaptation, distribution and reproduction in any medium or format, as long as you give appropriate credit to the original author(s) and the source, provide a link to the Creative Commons licence, and indicate if changes were made. The images or other third party material in this article are included in the article's Creative Commons licence, unless indicated otherwise in a credit line to the material. If material is not included in the article's Creative Commons licence and your intended use is not permitted by statutory regulation or exceeds the permitted use, you will need to obtain permission directly from the copyright holder. To view a copy of this licence, visit http://creativecommons.org/licenses/by/4.0/.

(C) The Author(s) 2021 